\title{
The Accuracy of Ultrasound-guided Fine-needle Aspiration and Core Needle Biopsy in Diagnosing Axillary Lymph Nodes in Women With Breast Cancer: a Systematic Review and Meta-Analysis
}

\section{Haining Zheng}

Peking University International Hospital

Rui Zhao

Peking University International Hospital

\section{Wei Wang}

Fourth Medical Center of PLA General Hospital

Xiaona Liu

Peking University International Hospital

\section{Xiaoqing Wang}

Peking University International Hospital

Chaoyang Wen ( $\nabla$ wenchaoyang_ycw@163.com )

Peking University International Hospital https://orcid.org/0000-0002-5234-8071

\section{Yubo Ren}

Peking University International Hospital

\section{Research}

Keywords: meta-analysis, fine-needle aspiration; core needle biopsy, axillary lymph nodes, diagnostic accuracy

Posted Date: March 6th, 2021

DOl: https://doi.org/10.21203/rs.3.rs-273798/v1

License: (c) (i) This work is licensed under a Creative Commons Attribution 4.0 International License. Read Full License 


\section{Abstract}

Background: This study performed an overall assessment of the accuracy of ultrasound-guided fineneedle aspiration (FNA) and core needle biopsy (CNB) for detecting axillary lymph nodes in women with breast cancer based on qualified studies.

Methods: Eligible studies and pertinent literature resources were identified on Cochrane, PubMed, Embase, CNKI, VIP, and Wanfang databases through searching key words or terms. The latest study was published in the March 2020. The eligible publications contained cohort and cross-sectional researches. All the publications obtained were tested for heterogeneity, and corresponding effect models were used to calculate amalgamative values of sensitivity, specificity and diagnostic odds ratio (DOR). Analysis of summary receiver operating characteristic (SROC) was performed on suspicious axillary lymph nodes.

Results: A total of 22 studies involving 3548 patients were included to explore the accuracy of FNA in identifying axillary lymph nodes in women with breast cancer. 11 studies involving 758 patients were included to explore the accuracy of CNB in identifying axillary lymph nodes in women with breast cancer. The accuracy of FNA in identifying suspicious axillary lymph nodes was specifically as follows: overall sensitivity was $79 \%$ (95\% Cl: 73\%-84\%), global specificity was $96 \%$ (95\% Cl: $92 \%-98 \%)$, overall positive likelihood ratio was 18.55 (95\% Cl: 10.53-32.69), overall negative likelihood ratio was 0.22 (95\% Cl: 0.17$0.28)$, the overall DOR was 71.68 (95\%Cl: 37.19-138.12), and the acreage under the SROC was (AUC = $0.94 ; 95 \% \mathrm{Cl}: 0.92-0.96)$. The accuracy of CNB in identifying suspicious axillary lymph nodes was specifically as follows: overall sensitivity was $85 \%$ (95\%Cl: $81 \%-89 \%)$, global specificity was $93 \%$ (95\% Cl: $87 \%-96 \%)$, overall positive likelihood ratio was 11.88 (95\% Cl: $6.56-21.50)$, overall negative likelihood ratio was 0.16 (95\% Cl: $0.12-0.21)$, the overall DOR was 66.83 (95\% Cl: $33.28-134.21)$, and the acreage under the SROC was (AUC $=0.96 ; 95 \% \mathrm{Cl}: 0.94-0.97)$.

Conclusions: The results indicated that both FNA and CNB had high accuracy for suspicious axillary lymph nodes.

\section{Background}

Axillary lymph node (ALN) metastasis is an important factor in clinical evaluation of prognosis of breast cancer. Conventional ALN dissection (ALND) plays a critical role in the staging of breast cancer, but it is also easy to cause serious postoperative complications and affect postoperative recovery. Sentinel lymph node biopsy (SLNB) is also applied in the assessment of ALN stage and the formulation of planning of treatment. However, SLNB surgery is highly traumatic, and requires accurate preoperative positioning and pathological diagnosis results, which is also prone to show false negative results. Therefore, developing a simple and effective diagnostic method is greatly important. Imaging examination as a non-invasive examination method is frequently applied in clinical diagnosis and treatment, and can help effectively diagnose ALN and avoid unnecessary SLNB. (1-4). 
Ultrasound is a commonly used imaging method to evaluate the properties of ALN. With its real-time dynamics, simple operation, and non-trauma, ultrasound can explore ALN from multiple angles and directions. In recent years, ultrasound-guided needle biopsy (UNB), contrast-enhanced ultrasound (CEUS) and elastography (UE) have enriched the content of ultrasonic diagnosis of ALN. Compared with conventional ultrasound, UNB used in combination with pathological biopsy results is more accurate and effective in the diagnosis of ALN metastasis (5-8). Based on qualified researches, this study aimed to evaluate the accuracy of FNA and CNB in finding suspicious axillary lymph nodes in women with breast cancer.

\section{Methods}

\section{Retrieve strategy}

All the eligible trails analyzing FNA and CNB in finding axillary lymph nodes in women with breast cancer were collected on Cochrane, PubMed, Embase, CNKI, VIP, and Wanfang databases. Other correlational studies or referenced data were retrieved as well. The latest study was published in March 2020. Two researchers independently retrieved there sources, with a third researcher to resolve any disagreement.

\section{Inclusion and exclusion criteria}

The criteria for inclusion: (1) cohort research or cross-sectional research; (2) suspicious axillary lymph nodes in women with breast cancer; (3) suspicious axillary lymph nodes were diagnostic by FNA or CNB; (4) the research offered the information of True-Positives(TP), False-Positive(FP), False-Negative(FN), True-Negative(TN); (5) written in English or Chinese.

The criteria for exclusion:(1) duplicate publications, or with the same contents and results; (2) case reports, theoretical studies, conference presentations, review literature, meta-analysis, expert commentary or analyses; (3) research without results relevant to this study; (4) without clinical outcomes of TP, FP, FN or TN; (5) Duplicate articles, or with the same contents and results.

Two researches decided whether the articles were included, with a third researcher to resolve any disagreement.

\section{Extraction data and quality evaluation}

Extraction of data was performed on all the publication included. Both essential information and main outcomes were extracted. For the essential information, writer's name, the capacity of sample, and the patients' age were included. For the clinical outcomes, a $2 \times 2$ diagnostic table was given for each included article, if the outcomes met the golden standard, FNA and CNB were entered as positive or negative. The data included True-Positive (TP), False-Positive (FP), False-Negative (FN) and TrueNegative (TN). If the value of a cell was 0 in the diagnostic table, 0.5 would be added to it for calculating availably. Sensitivity, specificity and likelihood ratio were computed. Accuracy of FNA and CAN in diagnosis was measured with the diagnostic odds ratio (DOR). When DOR equaled one, it suggested no 
distinguishing ability, with a higher value indicating a higher correlation of the evaluated diagnostic experiment. The above processes were independently performed by two researches, with a third researcher to resolve any disagreement.

\section{Statistical Analysis}

Stata 10.0 (TX, USA) was used for all statistical analyses. The results heterogeneity of trials present were determined based on the value of Chi-squared and $\mathrm{I}^{2}$ tests, according to which the analytical model fixed or random was chosen. If P-value of Chi-squared test was equal or lower than 0.05 and $\mathrm{I}^{2}$ test result was higher than $50 \%$, it suggested that heterogeneity was high and random effects model was chosen. If Pvalue of Chi-squared test was higher than 0.05 and $\mathrm{I}^{2}$ tests result was equal or lower than $50 \%$, heterogeneity was acceptable and fixed effects model was chosen. Based on the correlation analysis (Spearman's) between the logarithm of sensitivity and the logarithm of [1 - specificity], analysis of threshold evaluation was performed for further investigating heterogeneity. If a threshold effect existed, a negative correlation between sensitivity and specificity of the included articles (or a positive correlation between sensitivity and [1 -specificity]) was determined. A strong positive correlation between sensitivity and [1 - specificity] indicated effect of threshold. A curve plot of summary receiver operating characteristic (SROC) was given when the heterogeneity was caused by effect of threshold. The overestimated overall values of sensitivity and specificity were revealed by this method. Expect for analyzing the panel points of ROC, the SROC curve analysis was also used. The bias of publication was identified by Deeks' Funnel Asymmetry Plot.

\section{Results}

\section{Essential features of the included publications}

A total of 511 publications were identified by searching key words. After initial screening, 427 publications were excluded for reviewing title or abstract, and 84 publications were used for further assessment. 59 publications fail to meet the criteria of inclusion specifically as follows: theoretical research (6), without clinical outcomes (34), without comparative diagnostic method (19). At last 25 studies (9-34) with 4354 patients were included in this meta-analysis. The flow path is shown in Figure 1. Essential features of the researches, which included writer's name, the size of sample, the age, the value of TP, FP, FN, TN, were given in Table 1.

\section{Diagnostic accuracy of FNA}

A total of 22 studies with 3548 patients were included to explore the accuracy of FNA in identifying axillary lymph nodes in women with breast cancer. Random effects model was selected for merged analysis of DOR with high heterogeneity $\left(P<0.001, I^{2}=59.2 \%\right)$. The correlation result (Spearman's $R=-0.2331, P=0.2965)$ between the logarithm of sensitivity and the logarithm of [1 -specificity] indicated that there was no effect of threshold. 
The global sensitivity was $79 \%(95 \% \mathrm{Cl}$ : 73\%-84\%), and the global specificity was $96 \%(95 \% \mathrm{Cl}$ : $92 \%-98 \%)$. The overall positive and negative likelihood ratio were 18.55 (95\% Cl: 10.53-32.69), 0.22 (95\% Cl: 0.170.28 ), respectively. The first value suggested that FNA increased by 18.55 -fold of the odds in an effective diagnosis for suspicious axillary lymph nodes. Meanwhile, the second value showed that the FNA increased 0.22 for the false positive result. The overall DOR was 71.68 (95\% Cl: $37.19-138.12)$, and the odds were 71.68-fold higher for the positive FNA result among positive axillary lymph nodes when compared with negative axillary lymph nodes. The acreage under the SROC (AUC $=0.94 ; 95 \% \mathrm{Cl}$ : 0.920.96) was large. All the data were showed in Figure 2-5.

Based on the included and excluded criteria, 22 studies were enrolled in the meta-analysis of FNA. The quality evaluation and bias analysis were accessed by Deeks' Funnel Asymmetry Plot. The funnel graph for DOR of FNA of included publications was obviously symmetric, indicating a significant bias of publication (Figure 6, $\mathrm{P}=0.002$ ). The funnel plot revealed an apparent asymmetry, which suggested the presence of potential publication bias, language bias, and inflated estimates due to methodological design flaws in small studies, and/or lack of publication of small trials with opposite results.

\section{Diagnostic accuracy of CNB}

A total of 11 studies with 758 patients were included to explore the accuracy of CNB diagnosis in detecting axillary lymph nodes in women with breast cancer. Fix effects model was selected for merged analysis of DOR with low heterogeneity $\left(P=0.985, \mathrm{I}^{2}=0.0 \%\right)$. The correlation result (Spearman's $\mathrm{R}=-0.7963, \mathrm{P}=0.0034)$ between the logarithm of sensitivity and the logarithm of [1 - specificity] indicated that there was an effect of threshold.

The global sensitivity was $85 \%$ (95\% Cl: $81 \%-89 \%)$, and the global specificity was $93 \%$ (95\%Cl: $87 \%-96 \%)$. The overall positive and negative likelihood ratio were 11.88 (95\% Cl: 6.56-21.50), 0.16 (95\% Cl: $0.12-$ 0.21 ), respectively. The first value showed that FNA increased by 11.88 -fold of the odds in an effective diagnosis for suspicious axillary lymph nodes. Meanwhile, the second value showed that the FNA increased 0.16 for the false positive result. The overall DOR was 66.83 (95\% Cl: 33.28-134.21); the odds were 66.83-fold higher for the positive FNA result among positive axillary lymph nodes when compared with negative axillary lymph nodes. The acreage under the SROC (AUC $=0.96 ; 95 \% \mathrm{Cl}$ : 0.94-0.97) was large. Despite the threshold effect, the SROC curve $(A U C=0.96)$ indicated that the combined diagnosis was effective. All the data were showed in Figure 7-10.

Based on the included and excluded criteria, 11 studies were included into the meta-analysis of CNB. The quality evaluation and bias analysis were accessed by Deeks' Funnel Asymmetry Plot. The funnel graph for DOR of CNB in included publications was obviously symmetric, indicating no significant bias of publication (Figure 11, $\mathrm{P}=0.31$ ).

\section{Discussion}


The early stage of axillary lymph node metastasis of breast cancer is first implanted in the marginal lymph node sinus by lymphatic infusion, and then spread into the medullary sinus. At later stage, the lymph node is completely occupied by the cancer cells, and the cancer will continue to develop. Cancer cells break through the capsule and adhere to the surrounding tissues, accompanied by the proliferation of the surrounding interstitial fibrous tissues, resulting in poor mobility of the lymph nodes, a further increase in stiffness, less deformation by compression and an enlarged blue range in the elastogram. Clinical palpation (PE), mammography (MMG), ultrasonic imaging (US), computed tomography (CT), magnetic resonance imaging (MRI), positron emission tomography (PET), SLNB, FNA and CNB are used to determine axillary lymph node status. PE examines superficial lymph nodes but is not the means for detecting metastasis. MMG can only detect the anterior axillary lymph nodes and cannot completely cover the entire axillary lymph node area. CT clearly shows lymph nodes, but the radiation dose is large. MRI has a strong soft tissue resolution without radioactive damage, but the examination procedure is complicated, time-consuming and expensive. PET has a low spatial resolution and a certain false negative rate.

Histopathological examination after ALND is a reliable method for the diagnosis of axillary lymph node metastasis. However, ALND may cause many complications such as lymphatic reflux disorder, neuropathy and shoulder stiffness in the affected upper arm after operation, thus affecting the upper limb function of patients. SINB helps determine the nature of axillary lymph nodes in breast cancer, and ALND is not required for negative SLNB results. The false negative rate of SLNB is about $5-10 \%$, with less trauma and fewer complications, but there may also be local effusion, sensory nerve injury, lymphedema and other complications, and the occurrence of complications is closely related to the surgeon's proficiency.

Ultrasound-guided core needle biopsy (US-CNB) of breast masses is an accurate biopsy technique. Based on the histological results from puncture biopsy, the appropriate treatment for breast lesions can be clinically determined. Under the guidance of ultrasound, CNB and FNA aspiration cytology plays an important role in the diagnosis of breast lesions, so as to improve the early diagnosis rate of breast cancer and improve the prognosis of patients. FNA and CNB, which are the most widely used minimally invasive breast biopsy technologies, are mainly characterized by high accuracy, fast speed, small wound, few complications and low cost.

In this meta-analysis, we found that in the diagnosis of suspicious axillary lymph nodes, FNA had a sensitivity of $79 \%$ (95\% Cl: $73 \%-84 \%)$ and specificity of $96 \%$ (95\% Cl: $92 \%-98 \%)$; CNB had asensitivity of $85 \%$ (95\% Cl: $81 \%-89 \%)$ and specificity of $93 \%$ (95\% Cl: $87 \%-96 \%)$. The acreage under the SROC of FNA was (AUC $=0.94 ; 95 \% \mathrm{Cl}$ : 0.92-0.96), and the acreage under the SROC of CNB was (AUC $=0.96 ; 95 \% \mathrm{Cl}$ : 0.94-0.97). The results indicated that FNA and CNB had high diagnostic accuracy in detecting suspicious axillary lymph nodes.

However, there are certain limitations to the present analysis, which are as follows: (1) only English and Chinese articles were included; (2) the technique of the operator were different; (3) lesions can also affect 
the biopsy pathological results; (4) limited number of histological specimens may influence the accuracy of biopsy results; (5) only pooled data were analyzed, as individual patient data were not available, and this precluded more in-depth analyses.

\section{Conclusion}

The diagnostic accuracy of FNA in identifying suspicious axillary lymph nodes was specifically as follows: overall sensitivity was $79 \%(95 \% \mathrm{Cl}$ : $73 \%-84 \%)$, global specificity was $96 \%(95 \% \mathrm{Cl}$ : $92 \%-98 \%)$, the acreage under the SROC was (AUC $=0.94 ; 95 \% \mathrm{Cl}: 0.92-0.96$ ). The diagnostic accuracy of CNB for suspicious axillary lymph nodes was specifically as follows: overall sensitivity was $85 \%$ (95\% Cl: $81 \%-89 \%$ ), global specificity was $93 \%$ (95\% Cl: $87 \%-96 \%$ ), the acreage under the SROC was (AUC = 0.96; 95\% Cl: 0.94-0.97). Both FNA and CNB had a high diagnostic accuracy in identifying suspicious axillary lymph nodes, but the sensitivity, specificity and SROC of CNB was higher than FNA.

\section{Abbreviations}

$\mathrm{FNA}=$ fine-needle aspiration

CNB $=$ core needle biopsy

$\mathrm{DOR}=$ diagnostic odds ratio

SROC $=$ summary receiver operating characteristic

ALN= Axillary lymph node

ALND = Conventional ALN dissection

SLNB= Sentinel lymph node biopsy

UNB= ultrasound-guided needle biopsy

CEUS $=$ contrast-enhanced ultrasound

$\mathrm{UE}=$ elastography

$\mathrm{TN}=$ True-Negative

$\mathrm{TP}=$ True-Positive

$\mathrm{FP}=$ False-Positive

$\mathrm{FN}=$ False-Negative

$\mathrm{PE}=$ Clinical palpation 
$\mathrm{MMG}=$ mammography

US= ultrasonic imaging

$\mathrm{CT}=$ computed tomography

MRI= magnetic resonance imaging

$\mathrm{PET}=$ positron emission tomography

US-CNB= Ultrasound-guided core needle biopsy

\section{Declarations}

\section{Ethics approval and consent to participate}

No human or animals are involved in this research.

\section{Consent for publication}

Not applicable.

\section{Availiability of Data and Materials}

The analysed data sets generated during the study are available from the corresponding author on reasonable request.

\section{Competing interests}

The authors declare no conflicts of interest.

\section{Funding}

Not applicable

\section{Authors' contributions}

Substantial contributions to conception and design: HZ, RZ, WW

Data acquisition, data analysis and interpretation: $\mathrm{XL}, \mathrm{XW}, \mathrm{CW}, \mathrm{YR}$

Drafting the article or critically revising it for important intellectual content: $H Z, R Z, W W$

Final approval of the version to be published: All authors

Agreement to be accountable for all aspects of the work in ensuring that questions related to the accuracy or integrity of the work are appropriately investigated and resolved: All authors 
Acknowledgements

Not applicable

\section{References}

1. Arnedos M, Nerurkar A, Osin P, A R, apos, Hern, et al. Discordance between core needle biopsy (CNB) and excisional biopsy (EB) for estrogen receptor (ER), progesterone receptor (PgR) and HER2 status in early breast cancer (EBC). Annals of Oncology. 2009;20(12).

2. B S, M B, C M, S B. Retrospective preoperative assessment of the axillary lymph nodes in patients with breast cancer and literature review. Clinical radiology. 2015;70(9).

3. Wright AA, Zhang B, Keating NL, Weeks JC, Prigerson HG. Associations between palliative chemotherapy and adult cancer patients' end of life care and place of death: prospective cohort study. BMJ (Clinical research ed). 2014;348:g1219.

4. Zhao Y, Dong X, Li R, Song J, Zhang D. Correlation Between Clinical-Pathologic Factors and LongTerm Follow-Up in Young Breast Cancer Patients. Translational Oncology. 2015;8(4).

5. Bruening W, Fontanarosa J, Tipton K, Treadwell JR, Launders J, Schoelles K. Systematic review: comparative effectiveness of core-needle and open surgical biopsy to diagnose breast lesions. Annals of internal medicine. 2010;152(4):238-46.

6. D FA, A CJ, Christina C, B IO, N SP. Performance characteristics of ultrasound-guided fine-needle aspiration of axillary lymph nodes for metastatic breast cancer employing rapid on-site evaluation of adequacy: analysis of 136 cases and review of the literature. Cancer cytopathology. 2014;122(4).

7. Hafiz F, Choudhury T, Kamal M, Banu SG. The Spectrum of Pathological Changes in Breast Cancer Following Neoadjuvant Chemotherapy. Mymensingh Medical Journal. 2014;23(2):272-80.

8. Y Z, XQ D, RG L, X M, J S, YJ L, et al. Evaluation of the pathological response and prognosis following neoadjuvant chemotherapy in molecular subtypes of breast cancer. OncoTargets and Therapy. 2015;2015(default).

9. Ahn HS, Kim SM, Jang M, La Yun B, Kim SW, Kang E, et al. Comparison of sonography with sonographically guided fine-needle aspiration biopsy and core-needle biopsy for initial axillary staging of breast cancer. Journal of ultrasound in medicine : official journal of the American Institute of Ultrasound in Medicine. 2013;32(12):2177-84.

10. Boone BA, Huynh C, Spangler ML, Sumkin J, Johnson R, McGuire KP, et al. Axillary Lymph Node Burden in Invasive Breast Cancer: A Comparison of the Predictive Value of Ultrasound-Guided Needle Biopsy and Sentinel Lymph Node Biopsy. Clinical breast cancer. 2015;15(5):e243-8.

11. Ding; Y, Zhou; F, Hu; L, Liu; X, Qin. A. [Ultrasound-guided fine-needle aspiration biopsy combined with cytokeratin 19-fragments 21-1 in diagnosis of axillary lymph node metastasis in breast cancer]. Chin J Interv Imaging Ther. 2018;15(10):601-4.

12. Ganott MA, Zuley ML, Abrams GS, Lu AH, Kelly AE, Sumkin JH, et al. Ultrasound Guided Core Biopsy versus Fine Needle Aspiration for Evaluation of Axillary Lymphadenopathy in Patients with Breast 
Cancer. ISRN oncology. 2014;2014:703160.

13. Genta F, Zanon E, Camanni M, Deltetto F, Drogo M, Gallo R, et al. Cost/accuracy ratio analysis in breast cancer patients undergoing ultrasound-guided fine-needle aspiration cytology, sentinel node biopsy, and frozen section of node. World journal of surgery. 2007;31(6):1155-63.

14. Huo; L, Wu; K, Zhang; Y, Zhang; J, Gao. H. [Application of ultrasound-guided fine needle aspiration cytology in diagnosis of axillary lymph node metastasis in breast cancer]. Guangdong Medical Journal. 2016;37(S1):110-3.

15. Krishnamurthy S, Sneige N, Bedi DG, Edieken BS, Fornage BD, Kuerer HM, et al. Role of ultrasoundguided fine-needle aspiration of indeterminate and suspicious axillary lymph nodes in the initial staging of breast carcinoma. Cancer. 2002;95(5):982-8.

16. Le; J, Zhou; S, Hu; N, Gao; Y, Chen; M, Chang. C. [The Study of the Correlation between the Results of Ultrasound-guided Aspiration and Axillary Lymph Node Load in Breast Cancer]. Cinese J Ultrasound Med. 2017;33(12):1067-70.

17. Liu; M, Liu; P, Xie; F, Yang. D. [Ultrasound-guided fine-needle aspiration for the evaluation of axillary lymph node metastasis in breast cancer]. Chin J Gen Surg. 2011;26(1):25-8.

18. Maxwell AJ, Bundred NJ, Harvey J, Hunt R, Morris J, Lim YY. A randomised pilot study comparing 13 $\mathrm{G}$ vacuum-assisted biopsy and conventional $14 \mathrm{G}$ core needle biopsy of axillary lymph nodes in women with breast cancer. Clinical radiology. 2016;71(6):551-7.

19. Nakamura R, Yamamoto N, Miyaki T, Itami M, Shina N, Ohtsuka M. Impact of sentinel lymph node biopsy by ultrasound-guided core needle biopsy for patients with suspicious node positive breast cancer. Breast cancer (Tokyo, Japan). 2018;25(1):86-93.

20. Popli MB, Sahoo M, Mehrotra N, Choudhury M, Kumar A, Pathania OP, et al. Preoperative ultrasoundguided fine-needle aspiration cytology for axillary staging in breast carcinoma. Australasian radiology. 2006;50(2):122-6.

21. Rao R, Lilley L, Andrews V, Radford L, Ulissey M. Axillary staging by percutaneous biopsy: sensitivity of fine-needle aspiration versus core needle biopsy. Annals of surgical oncology. 2009;16(5):1170-5.

22. Rautiainen $S$, Masarwah A, Sudah M, Sutela A, Pelkonen $O$, Joukainen $S$, et al. Axillary lymph node biopsy in newly diagnosed invasive breast cancer: comparative accuracy of fine-needle aspiration biopsy versus core-needle biopsy. Radiology. 2013;269(1):54-60.

23. Ruan; Y, Pan; D, HangYanzhu; Shen; Z, Zheng. L. [The diagnostic value of ultrasound-guided fine needle aspiration of axillary lymph node metastasis in breast cancer ]. Chin J Prev Contr Chron Dis. 2018;26(10):763-6.

24. Sang; G, He; Y, Lu; C, Sun. R. [Utilization of preoperative ultrasound-guided fine needle aspiration cytology for the evaluation of axillary lymph nodes status in patients with early stage breast cancer]. Chin J Endocr Surg. 2019;13(3):241-4.

25. Sang; Y, Xie. H. [Application value of ultrasound-guided fine-needle and core-needle aspiration biopsy for axillary staging of breast cancer]. Guangxi Medical Journal. 2016;38(11):1544-6. 
26. Topal U, Punar S, Tasdelen I, Adim SB. Role of ultrasound-guided core needle biopsy of axillary lymph nodes in the initial staging of breast carcinoma. European journal of radiology. 2005;56(3):382-5.

27. van Wely BJ, de Wilt JH, Schout PJ, Kooistra B, Wauters CA, Venderinck D, et al. Ultrasound-guided fine-needle aspiration of suspicious nodes in breast cancer patients; selecting patients with extensive nodal involvement. Breast cancer research and treatment. 2013;140(1):113-8.

28. Vidya R, lqbal FM, Bickley B. Pre-operative axillary staging: should core biopsy be preferred to fine needle aspiration cytology? Ecancermedicalscience. 2017;11:724.

29. Wang; J, Wang; W, Wei; C, Gu. Z. [Diagnosis of axillary lymph node metastasis in breast cancer by ultrasound-guided needle biopsy]. J Chin Ultrasound in Med. 2013;15(3):203-4.

30. Wang; Y, Gao; W, Yin; S, Zhang; L, Zhang; H, Yan; K, et al. [The diagnostic value of ultrasound and ultrasound-guided needle biopsy for axillary lymph nodes in breast cancer]. Chin J Gen Surg. 2005;20(5):322-3.

31. Wei; Y, Li; Y, Zhang; Q, Jiang. D. [The value of preoperative ultrasound guide fine needle aspiration for axillary lymph node metastasis in breast canser]. Modern Oncology. 2014;22(03):569-71.

32. Yu; C, Chen; X, Fan; Q, Chen; J, Wang; J, Ling. Z. [A retrospective study on the effect of ultrasoundguided core needle biopsy in breast cancer patients after neoadjuvant chemotherapy]. JOURNAL OF HARBIN MEDICAL UNIVERSITY. 2018;52(05):491-5.

33. Zhao; Y, Bai; L, Tang; Y, Liu; W, Hao; X, Chen; C, et al. [Clinical value of ultrasound-guided core needle biopsy for detecting axillary lymph node metastasis in breast cancer]. Mil Med Sci. 2015;39(07):5415.

34. Zhou; S, Le; J, Hu; N, Gao; Y, Chang. C. The study of ultrasound guided fine needle aspiration of axillary lymph nodes in breast cancer. Chin J Ultrasonogr. 2017;26(6):527-30.

\section{Tables}

Table 1. The basic characteristics description of included studies 


\begin{tabular}{|c|c|c|c|c|c|c|c|}
\hline Study & Sample & Age & Diagnostic Method & TP & $\mathrm{FP}$ & $\mathrm{FN}$ & $\mathrm{TN}$ \\
\hline Rikiya Nakamura 2017 a & 487 & 54 & FNA & 367 & 1 & 199 & 96 \\
\hline Rikiya Nakamura 2017 b & 172 & 56 & CNB & 214 & 0 & 30 & 12 \\
\hline Raghavan Vidya 2017 a & 43 & - & FNA & 18 & 0 & 7 & 25 \\
\hline Raghavan Vidya 2017 b & 38 & - & CNB & 27 & 0 & 0 & 11 \\
\hline Roshni Rao 2009 a & 22 & 50.5 & FNA & 12 & 0 & 4 & 6 \\
\hline Roshni Rao 2009 b & 25 & 52.5 & CNB & 18 & 0 & 4 & 3 \\
\hline Marie A. Ganott 2014 a & 95 & - & FNA & 55 & 0 & 10 & 5 \\
\hline Marie A. Ganott 2014 b & 95 & - & CNB & 61 & 0 & 4 & 5 \\
\hline Hye Shin Ahn 2013 a & 48 & 49 & FNA & 19 & 0 & 7 & 22 \\
\hline Hye Shin Ahn 2013 b & 48 & 49 & CNB & 20 & 0 & 6 & 22 \\
\hline Suvi Rautiainen 2013 a & 178 & 61.4 & FNA & 37 & 0 & 14 & 15 \\
\hline Suvi Rautiainen 2013 b & 178 & 61.4 & CNB & 45 & 0 & 9 & 15 \\
\hline A.J. Maxwell 2016 & 40 & 57 & CNB & 15 & 0 & 4 & 18 \\
\hline B. J. van Wely 2013 & 199 & - & FNA & 157 & 0 & 19 & 22 \\
\hline Franco Genta 2007 & 370 & - & FNA & 43 & 0 & 23 & 31 \\
\hline MB Popli 2006 & 24 & - & FNA & 15 & 0 & 4 & 5 \\
\hline U־gur Topal 2005 & 39 & - & CNB & 30 & 0 & 3 & 6 \\
\hline Savitri Krishnamurthy 2002 & 103 & - & FNA & 51 & 16 & 12 & 24 \\
\hline Yanbin Wang 2005 & 86 & 48.9 & FNA & 50 & 0 & 5 & 31 \\
\hline Yan Ding 2018 & 148 & 49.2 & FNA & 64 & 0 & 17 & 75 \\
\hline Shichong Zhou 2017 & 500 & 45.2 & FNA & 192 & 0 & 54 & 136 \\
\hline Jian Le 2017 & 255 & 52 & FNA & 105 & 3 & 24 & 123 \\
\hline Juan Wang 2013 & 87 & 51.2 & FNA & 60 & 0 & 4 & 20 \\
\hline Leijun Huo 2016 & 89 & 46.29 & FNA & 19 & 1 & 15 & 8 \\
\hline Ying Sang 2016 a & 48 & 49 & FNA & 19 & 0 & 7 & 22 \\
\hline Ying Sang 2016 b & 48 & 49 & CNB & 20 & 0 & 6 & 22 \\
\hline Chunyang Yu 2018 & 27 & 47 & CNB & 35 & 1 & 2 & 3 \\
\hline Guo Sang 2019 & 58 & 48.3 & FNA & 37 & 0 & 7 & 14 \\
\hline
\end{tabular}




\begin{tabular}{|llllllll|} 
Yibo Zhao 2015 & 454 & 49 & FNA & 25 & 0 & 14 & 61 \\
\hline Miao Liu 2011 & 40 & 52 & FNA & 12 & 0 & 11 & 17 \\
\hline Yuntao Wei 2014 & 47 & 52.2 & FNA & 24 & 5 & 0 & 18 \\
\hline Yajun Ruan 2018 & 167 & 53.2 & FNA & 73 & 1 & 2 & 20 \\
\hline
\end{tabular}

FNA: fine-needle aspiration; CNB: core needle biopsy; TP: true positive, FP: false positive; FN: false negative; TN: true negative.

a: patients diagnosed by FNA, b: patients diagnosed by CNB.

\section{Figures}

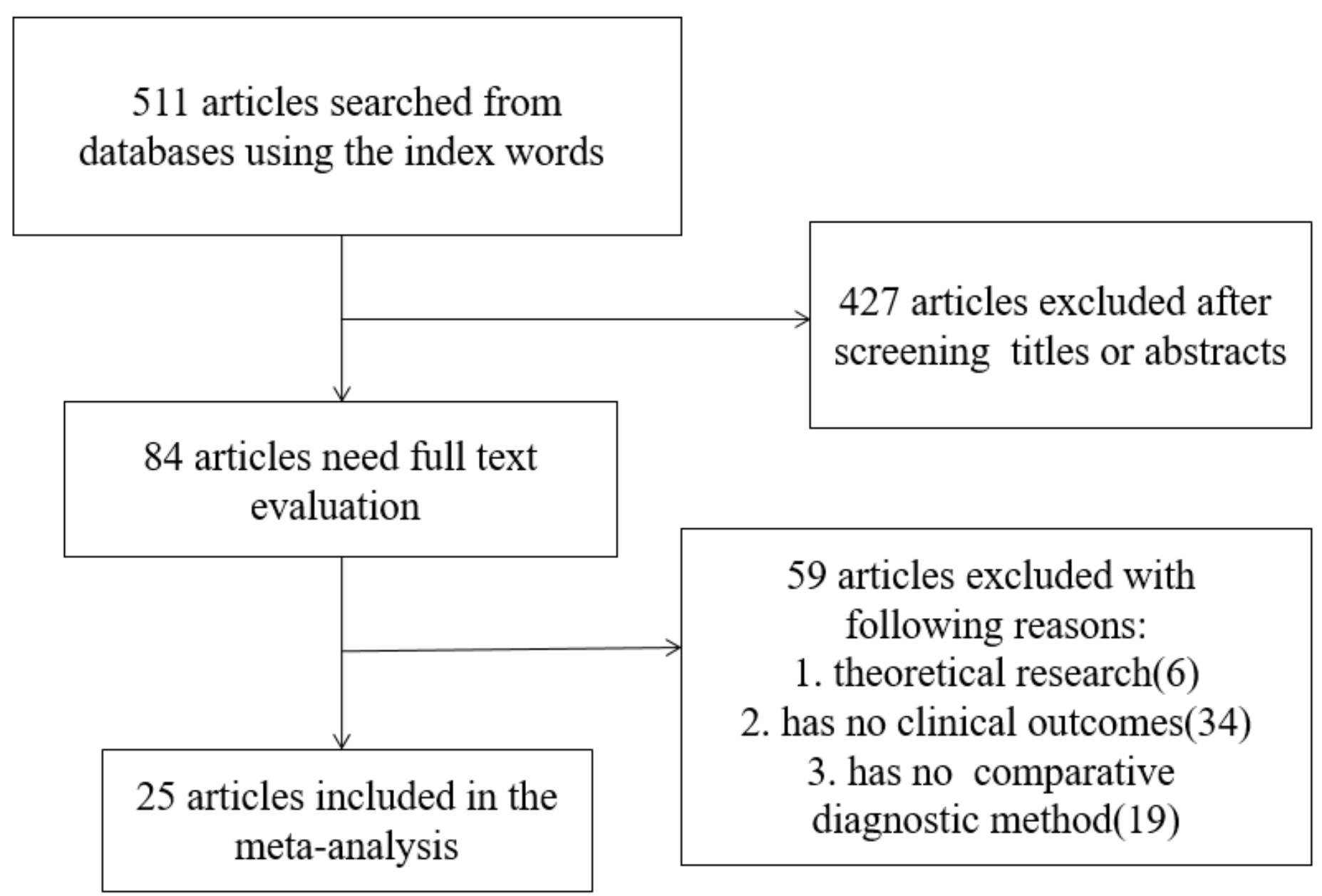

Figure 1

Flow diagram of the literature search and selection process 


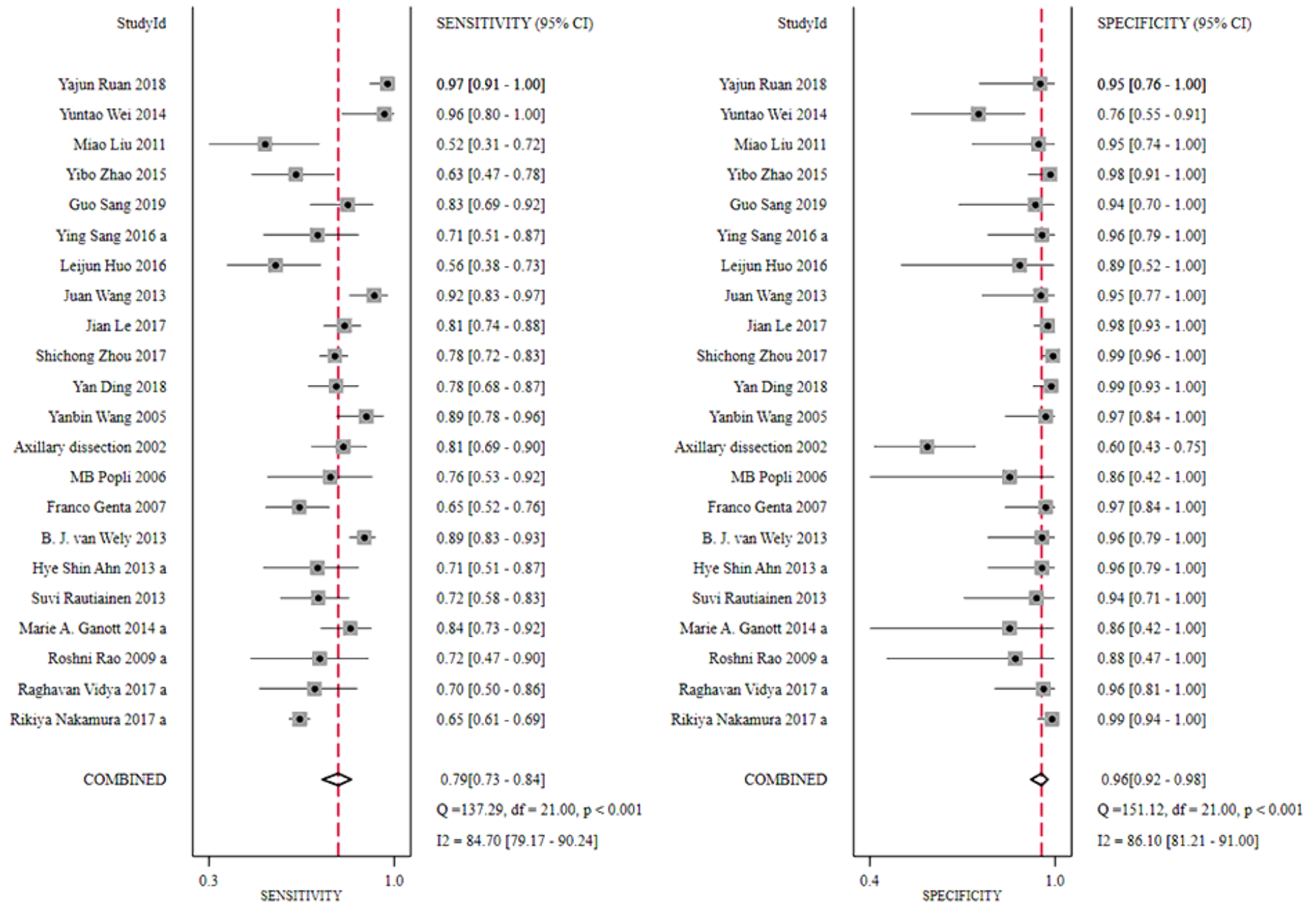

Figure 2

Forest plot showing the sensitivity and specificity values of FNA for suspicious axillary lymph nodes 


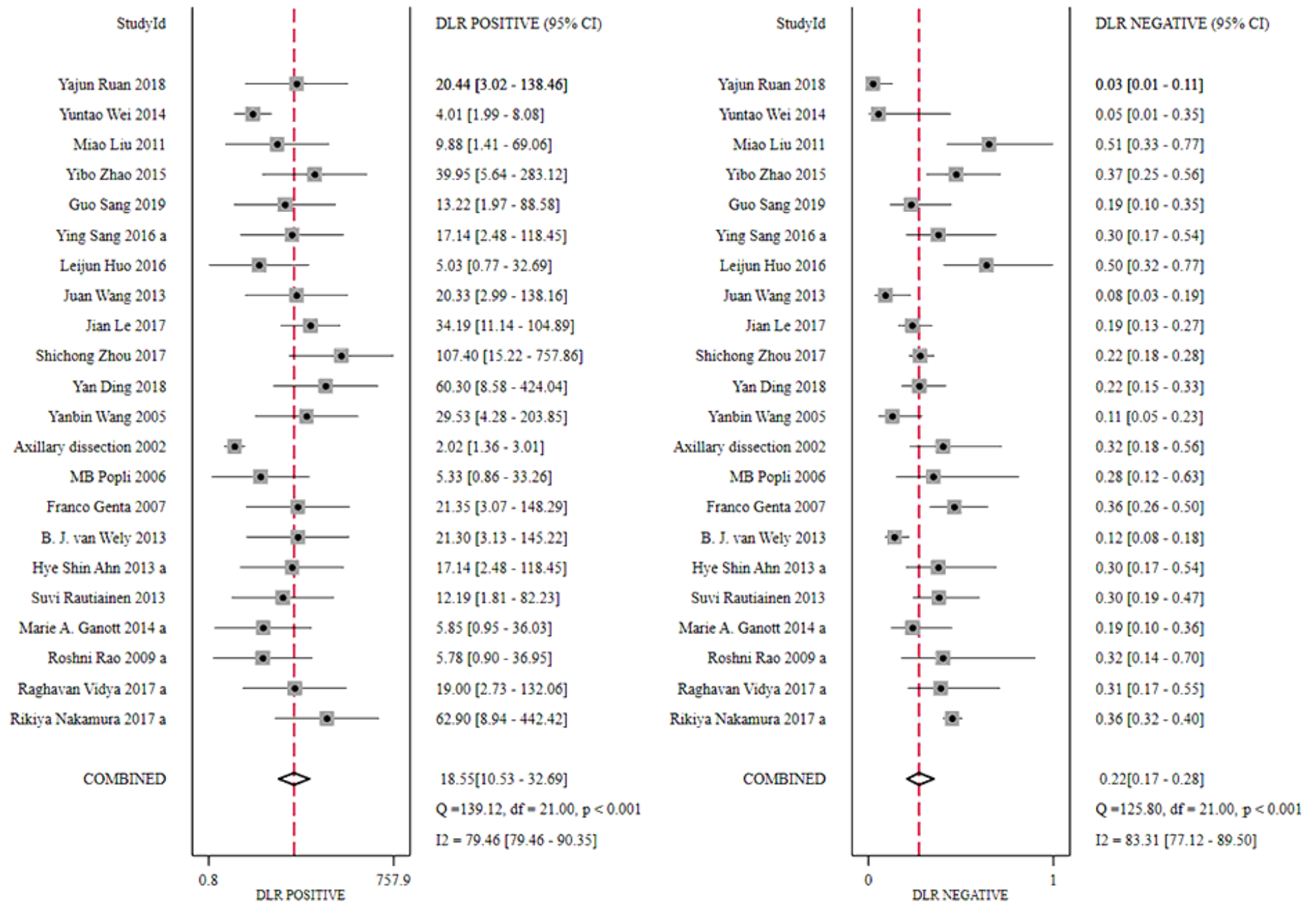

Figure 3

Forest plot showing the positive and negative likelihood ratio of FNA for suspicious axillary lymph nodes 


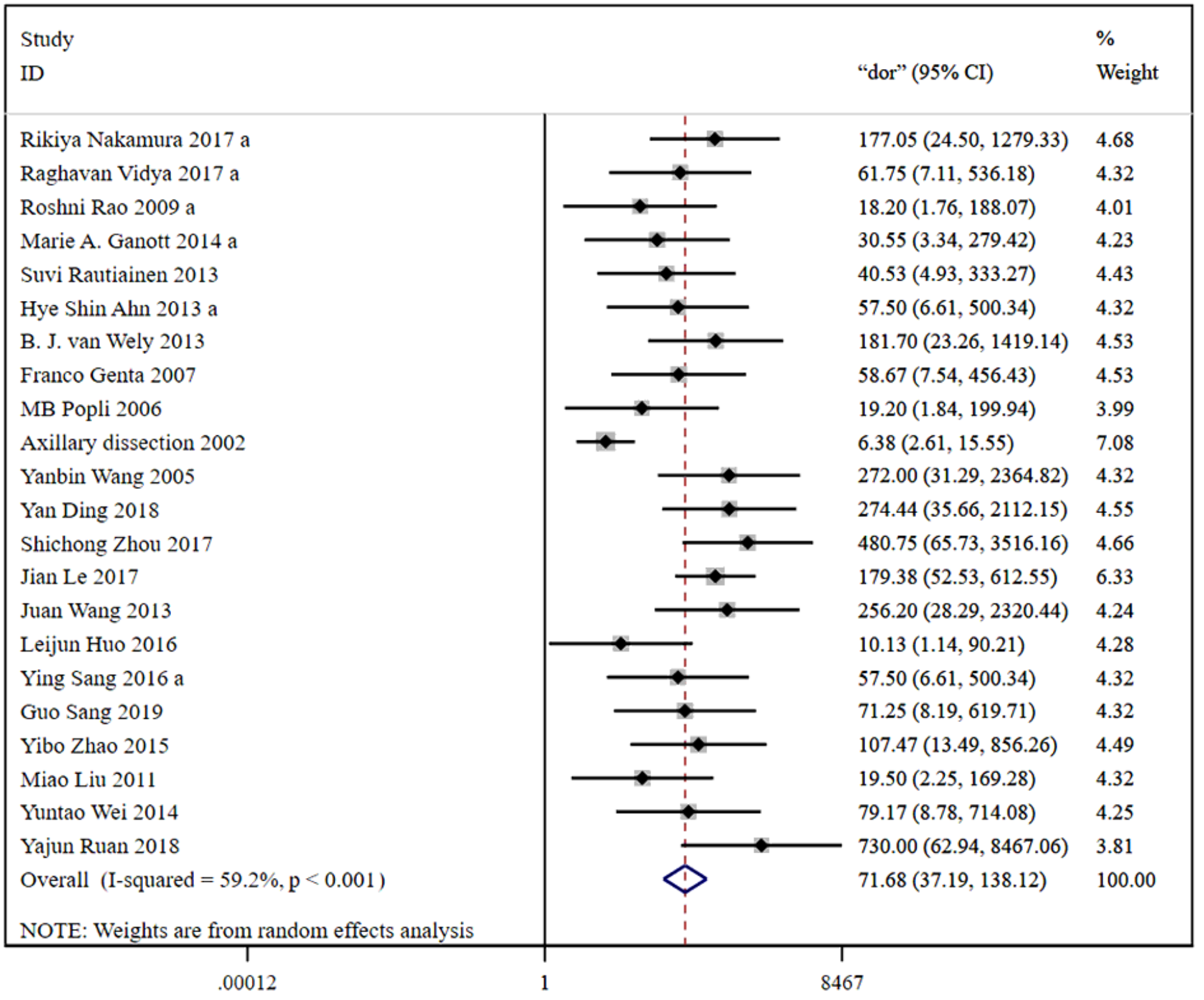

Figure 4

Forest plot showing the diagnostic odds ratio of FNA for suspicious axillary lymph nodes 


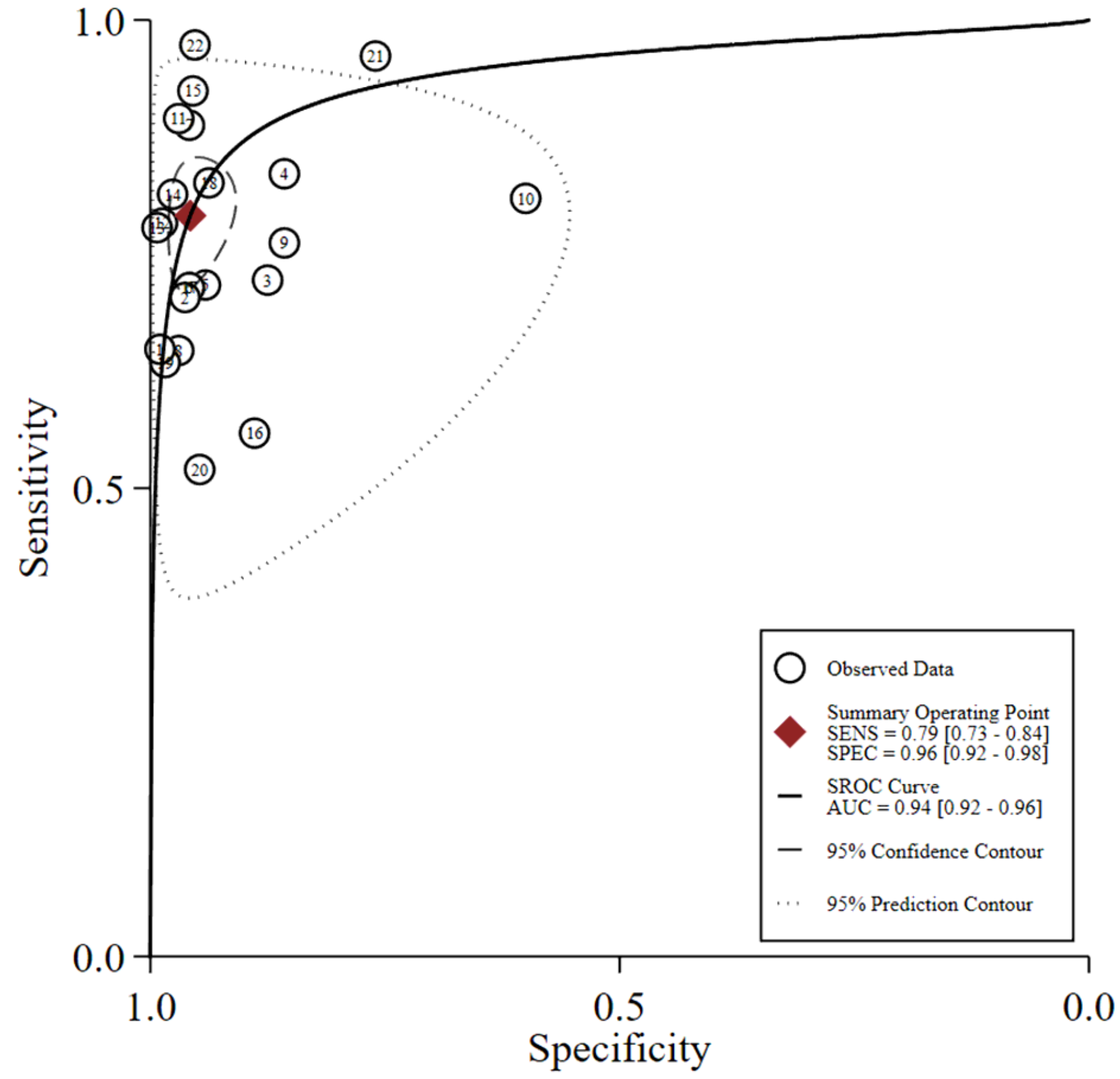

Figure 5

Summary ROC plots for diagnostic accuracy of FNA for suspicious axillary lymph nodes 


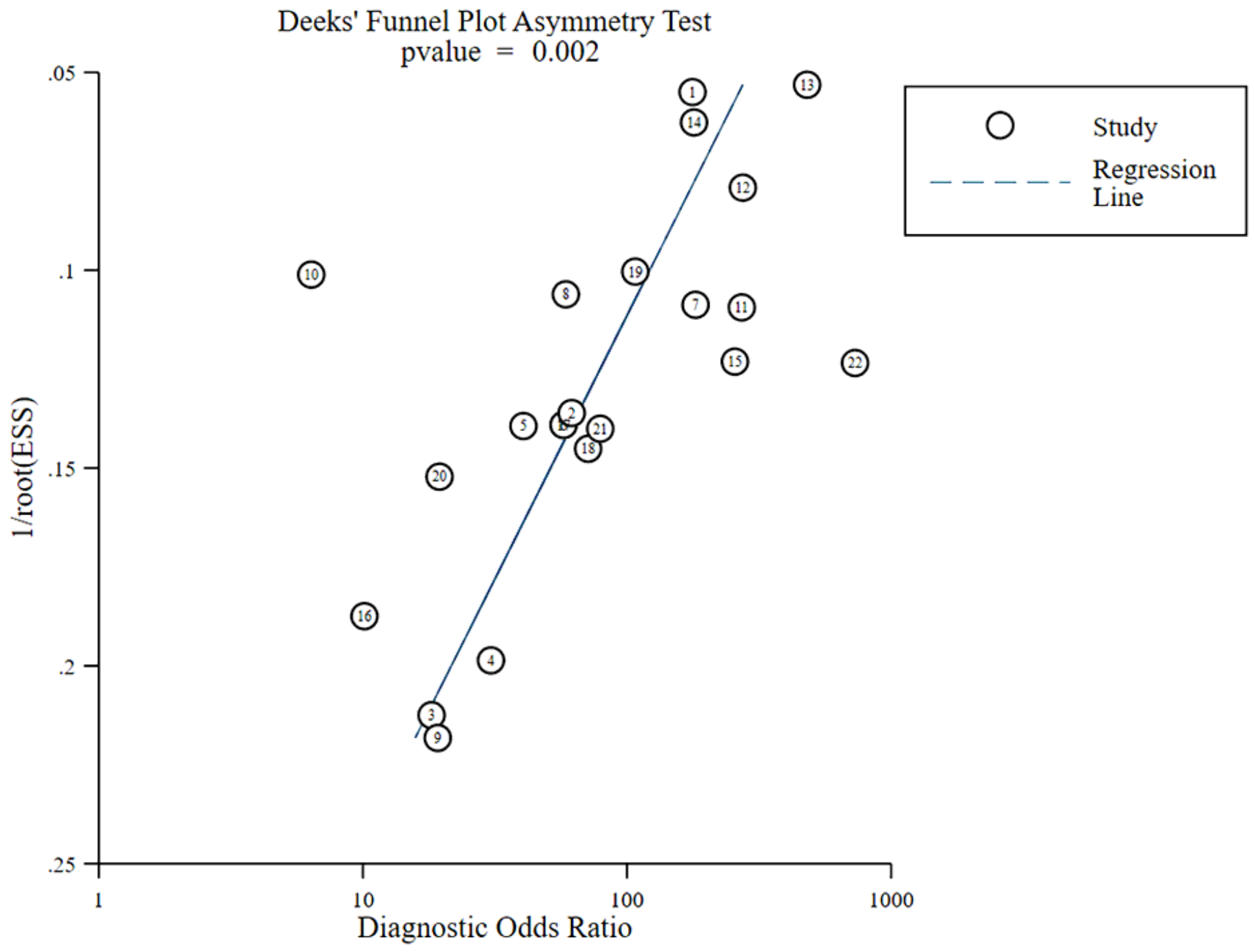

Figure 6

Funnel plot of studies about FNA included in the meta-analysis 


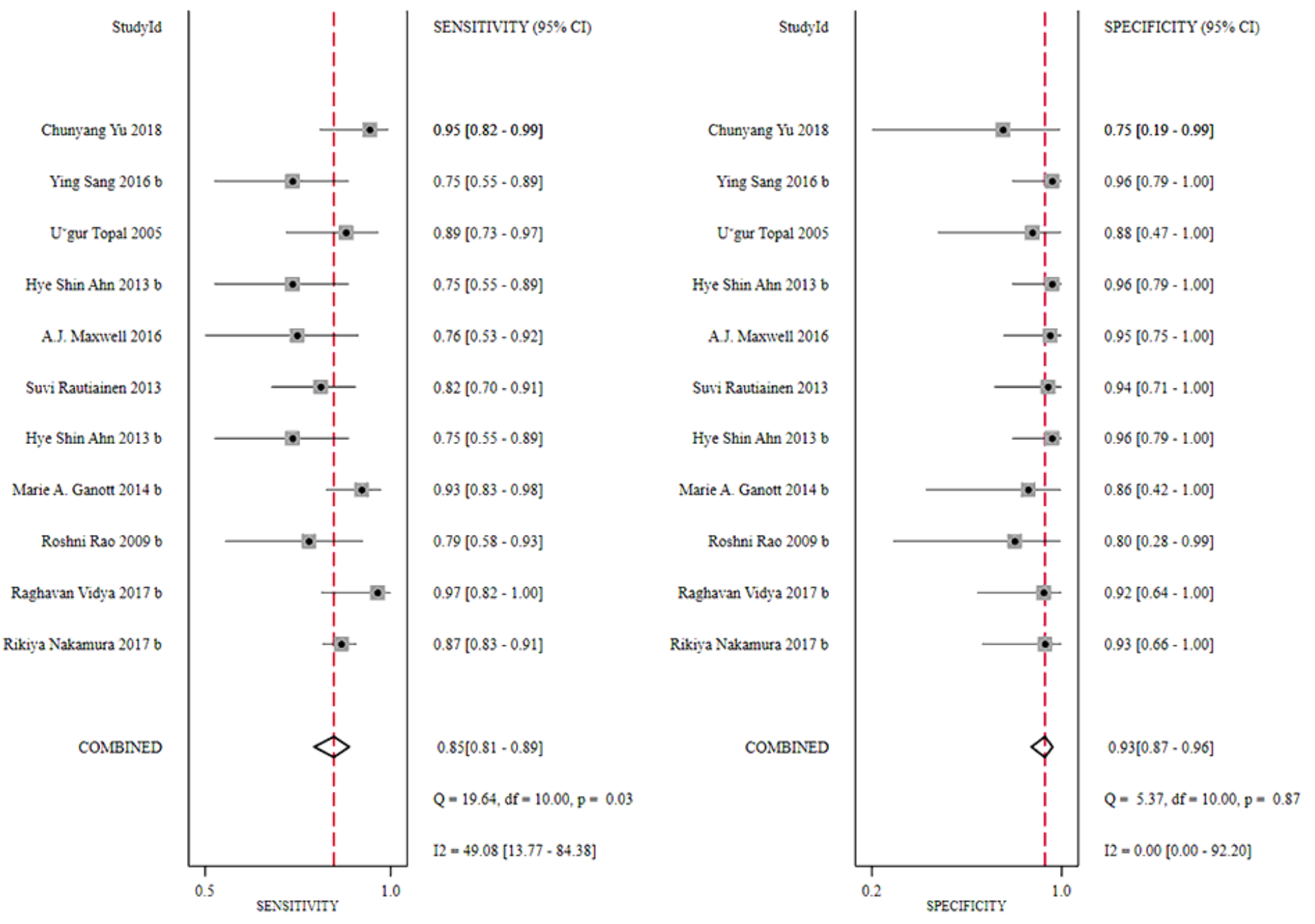

\section{Figure 7}

Forest plot showing the sensitivity and specificity values of CNB for suspicious axillary lymph nodes 


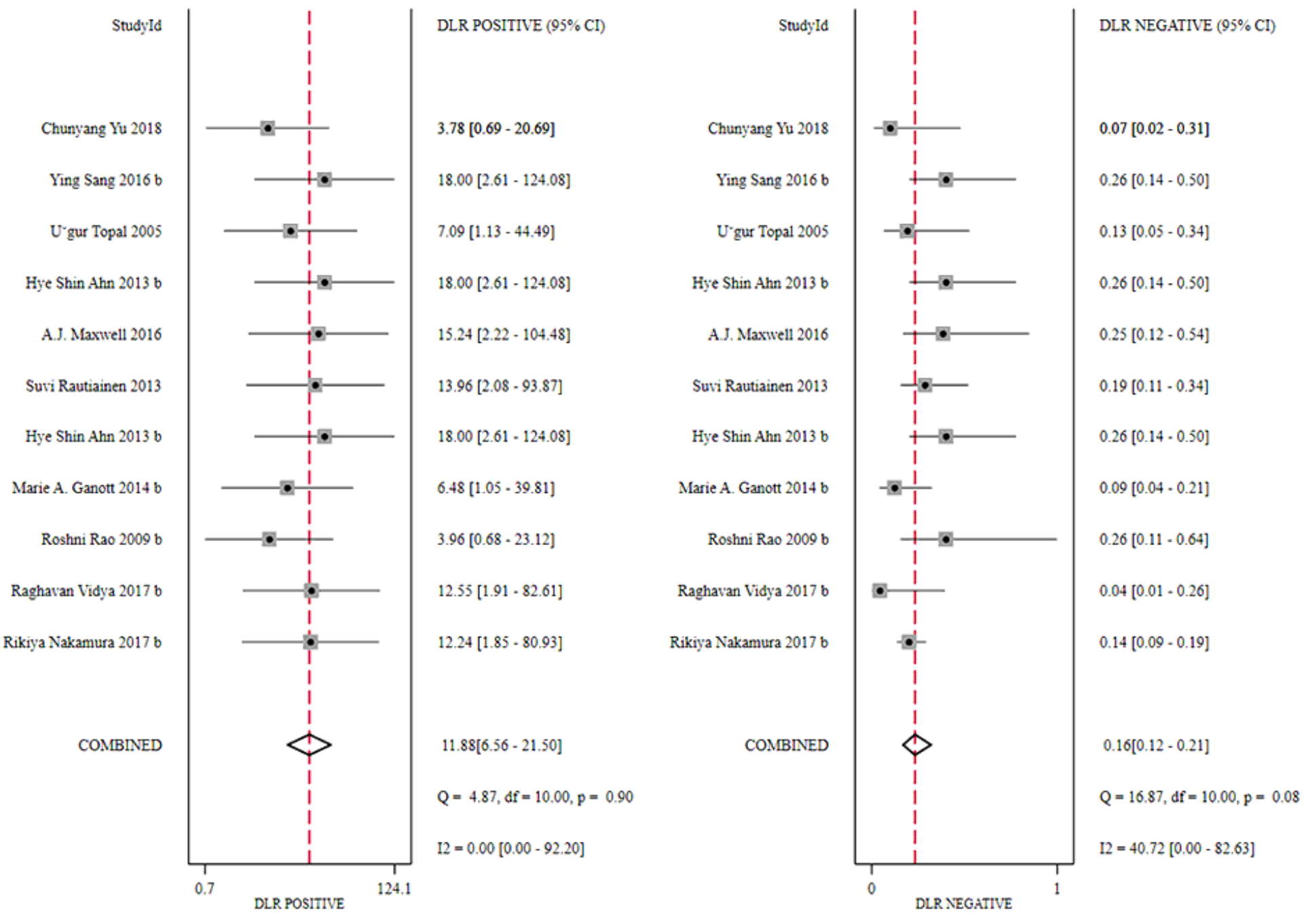

Figure 8

Forest plot showing the positive and negative likelihood ratio of CNB for suspicious axillary lymph nodes 
ID

"dor" $(95 \%$ CI)

Weight

Rikiya Nakamura 2017 b

Raghavan Vidya 2017 b

Roshni Rao 2009 b

Marie A. Ganott 2014 b

Hye Shin Ahn 2013 b

Suvi Rautiainen 2013

A.J. Maxwell 2016

Hye Shin Ahn 2013 b

Ǔgur Topal 2005

Ying Sang 2016 b

Chunyang Yu 2018

Overall (I-squared $=0.0 \%, \mathrm{p}=0.985$ )

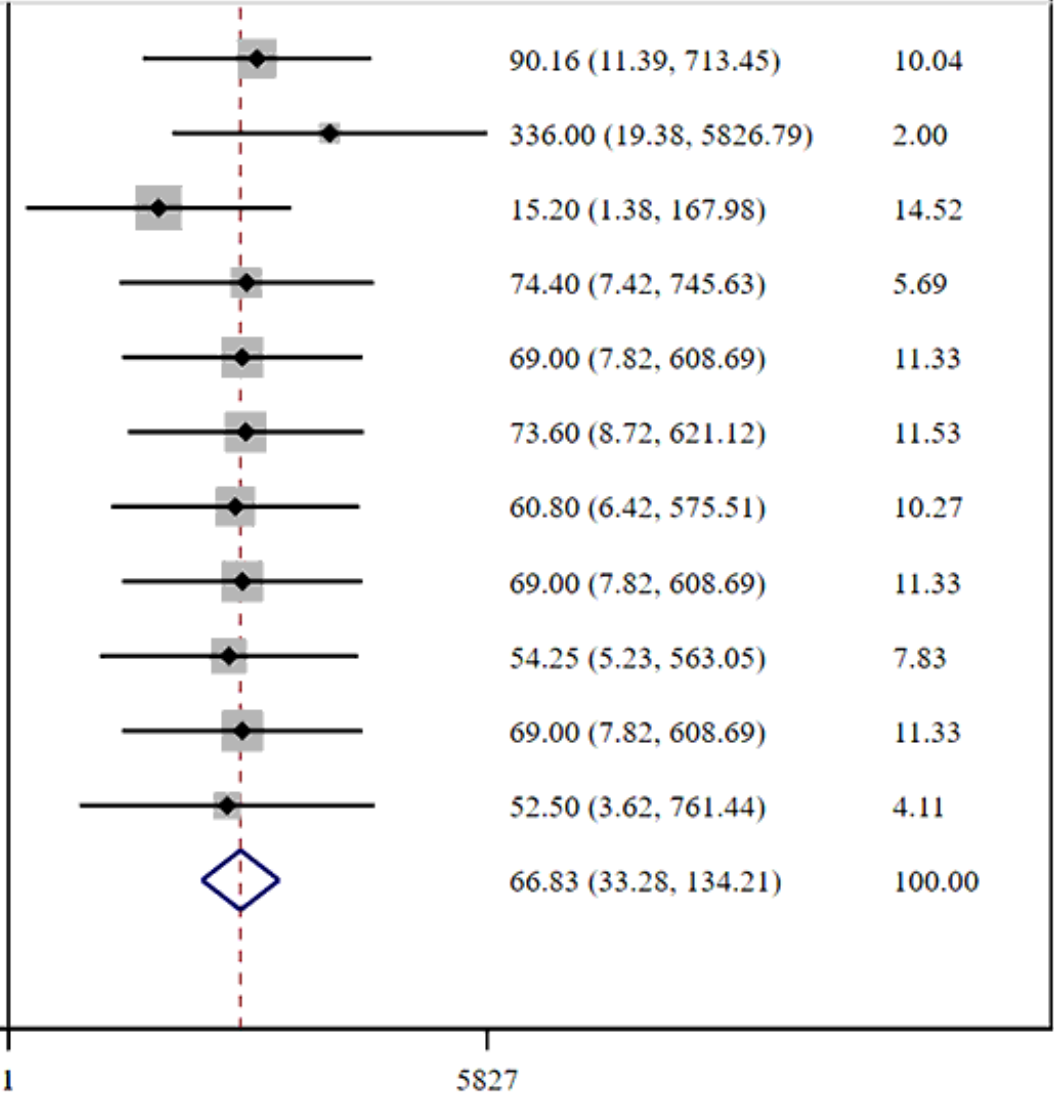

\section{Figure 9}

Forest plot showing the diagnostic odds ratio of CNB for suspicious axillary lymph nodes 


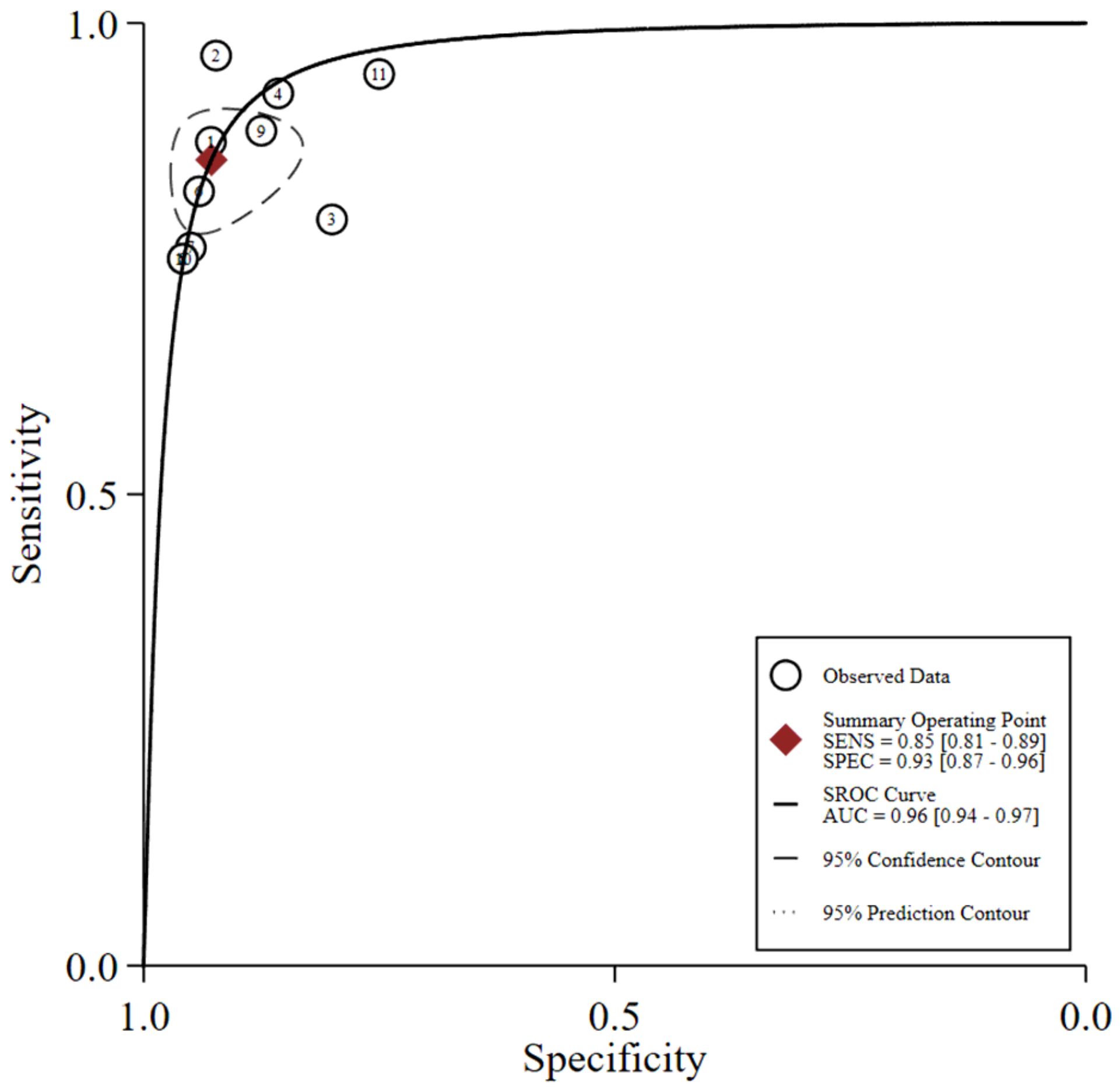

Figure 10

Summary ROC plots for diagnostic accuracy of CNB for suspicious axillary lymph nodes 


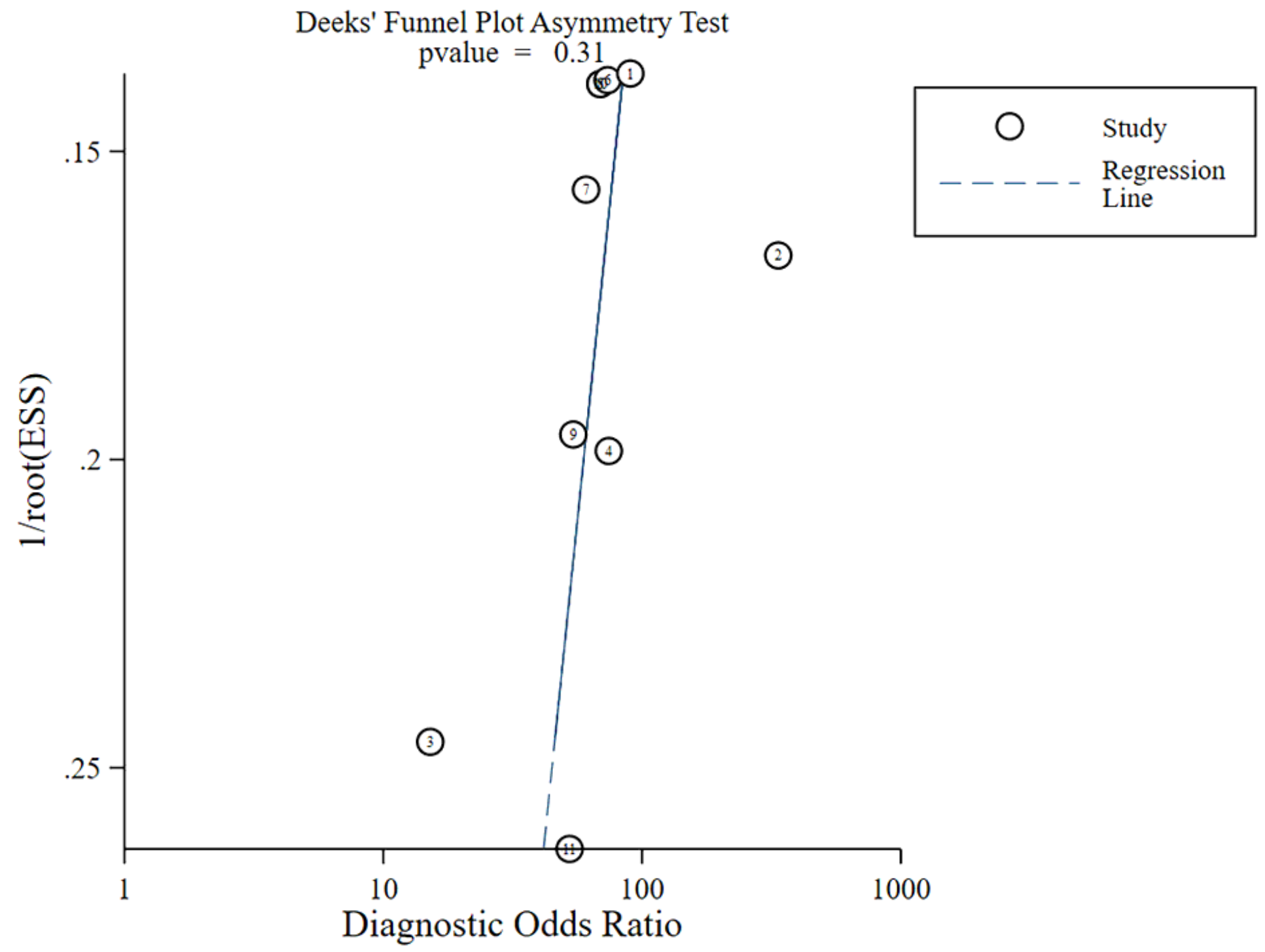

Figure 11

Funnel plot of studies about CNB included in the meta-analysis 